\title{
Clinical characteristics of non-B non-C hepatocellular carcinoma: a single-center retrospective study.
}

\author{
$\operatorname{AUTHOR}(\mathrm{S}):$ \\ Kim, Soo Ki; Marusawa, Hiroyuki; Eso, Yuji; Nishikawa, \\ Hiroki; Ueda, Yoshihide; Kita, Ryuichi; Kimura, Toru; Chiba, \\ Tsutomu; Osaki, Yukio; Kudo, Masatoshi
}

\section{CITATION:}

Kim, Soo Ki ... [et al]. Clinical characteristics of non-B non-C hepatocellular carcinoma: a single-center retrospective study.. Digestion 2011, 84 Suppl 1: 43-49

\section{ISSUE DATE:}

2011-12

URL:

http://hdl.handle.net/2433/152377

\section{RIGHT:}

(c) 2011 S. Karger AG, Basel; This is not the published version. Please cite only the published version.; この論文は出版社版でありません。引用の 際には出版社版をご確認ご利用ください。 
Clinical characteristics of nonBnonC-hepatocellular carcinoma : A single center retrospective study.

Soo Ki Kim ${ }^{1,2}$, Hiroyuki Marusawa ${ }^{1}$, Yuji Eso ${ }^{1,2}$, Hiroki Nishikawa ${ }^{2}$, Yoshihide Ueda ${ }^{1}$, Ryuichi Kita $^{2}$, Toru Kimura ${ }^{2}$, Tsutomu Chiba ${ }^{1}$, Yukio Osaki², Masatoshi Kudo ${ }^{3}$

1. Department of Gastroenterology and Hepatology, Graduate School of Medicine, Kyoto University

2. Department of Gastroenterology and Hepatology, Osaka Red Cross Hospital

3. Department of Gastroenterology and Hepatology, Kinki University School of Medicine

short title: Clinical characteristics of nonBnonC-hepatocellular carcinoma

Corresponding author: Hiroyuki Marusawa, M.D., Ph.D.

Department of Gastroenterology and Hepatology,

Graduate School of Medicine, Kyoto University,

Shogoin-Kawaharacho 54, Sakyo-ku, Kyoto, 606-8507, Japan.

E-mail: maru@kuhp.kyoto-u.ac.jp

Telephone number: $+81-75-751-4319$

Fax number: $+81-75-751-4303$

Key words: liver cancer, nonBnonC-HCC, nonalcoholic steatohepatitis, alcohol, diabetes

Conflicts of interest: Conflicts of interest: All authors disclose no conflicts. 


\begin{abstract}
Background/Aims: To clarify risk factors and clinical features of both hepatitis B surface antigen and anti-HCV negative hepatocellular carcinoma (NBNC-HCC).

Methods: HCC patients ( $\mathrm{n}=1109)$ diagnosed at a single center were categorized based on the presence of serum HBsAg and HCVAb. Clinical characteristics of 127 NBNC-HCC patients were evaluated.
\end{abstract}

Results: NBNC-HCC patients were stratified as those with alcoholic liver disease (ALD-HCC, $\mathrm{n}=42$ ) and alcohol unrelated liver disease (nonALD-HCC, $\mathrm{n}=85$ ). Compared with the ALD-HCC group, the nonALD-HCC group had a higher prevalence of diabetes $(\mathrm{P}=0.015)$, larger tumor size $(\mathrm{P}=0.007)$, and higher tumor marker levels $(\mathrm{P}=0.014)$. Liver function results were significantly worse in ALD-HCC than in nonALD-HCC. Although the ALD-HCC group had a higher tendency toward recurrence than the nonALD-HCC group, survival rates were similar between groups $(\mathrm{P}=0.352)$.

Conclusion: Alcohol consumption was the most common etiologic factor for NBNC-HCC, and diabetes may be related to the development of HCC in nonALD-HCC patients. NonALD-HCC tended to be diagnosed at a more advanced stage, whereas liver function was worse, and tumor recurrence rate was higher in ALD-HCC patients. Further examination of the risk factors and establishment of a precise surveillance system are necessary for early diagnosis and the development of curative therapies for NBNC-HCC. 


\section{Introduction}

Hepatocellular carcinoma (HCC) is one of the most common causes of cancer-related deaths worldwide $[1,2]$. In Japan, chronic hepatitis C virus (HCV) infection is considered to the most significant risk factor for the development of $\mathrm{HCC}$, and the second-most important factor is hepatitis B virus (HBV) infection. Based on a report by the Liver Cancer Study Group of Japan, approximately $15 \%$ of HCC patients in Japan are hepatitis B surface antigen (HBsAg) positive (B-HCC) and 70\% are anti-HCV (HCVAb) positive (C-HCC) [3]. Recent progress in the management of patients with viral hepatitis by specific anti-viral therapy, including interferon and nucleotide analogues, however, has led to better prevention of cancer development and improved disease prognosis [4-7]. On the other hand, the number of patients that are both HBsAg- and HCVAb-negative HCC (NonB-NonC HCC [NBNC-HCC]) has increased and NBNC-HCC is reported to account for $12 \%$ to $20 \%$ of all HCC cases in Japan $[3,8]$.

NBNC-HCC is considered to be associated with several etiologic factors such as alcoholic liver injury, autoimmune hepatitis $(\mathrm{AIH})$, primary biliary cirrhosis (PBC), primary sclerosing cholangitis, and nonalcoholic fatty liver disease (NAFLD)/nonalcoholic steatohepatitis (NASH). In addition, a variety of clinical factors are also involved in the development and progression of NBNC-HCC, including age, sex, alcohol consumption, and diabetes mellitus $[9,10]$. There are only a few reports, however, on the clinical characteristics of NBNC-HCC and the actual state of NBNC-HCC has not been fully elucidated [3,8,11,12].

Due to the lack of understanding of the clinical features of NBNC-HCC, neither early detection nor improvement of prognosis has been achieved in patients with NBNC-HCC. Therefore, it is important to understand the complex interactions of the risk factors and clinical 
features of NBNC-HCC. In this study, we aimed to clarify the clinical characteristics of NBNC-HCC and discuss the etiology of NBNC-HCC.

\section{Methods}

\section{Patients}

A total of 1109 HCC patients diagnosed at Osaka Red Cross Hospital from April 1, 2004 to March 31, 2010 were enrolled in the study. The diagnosis of HCC was made based on the presence of both characteristic imaging findings and increases in serum tumor markers; the diagnostic criteria for HCC via imaging was based on previous reports of hyperattenuation at the arterial phase and hypoattenuation at the portal phase in the tumor, determined by dynamic computed tomography and/or magnetic resonance imaging [13]. In addition, increases in serum tumor markers such as $\alpha$-fetoprotein, des- $\gamma$-carboxy prothrombin, or Lens culinaris agglutinin-reactive fraction of $\alpha$-fetoprotein, were also required for the diagnosis of HCC. In doubtful cases, the diagnosis was confirmed by pathologic findings based on liver biopsies obtained under ultrasound guidance.

\section{Classification of HCC according to etiology}

The presence of serum HBsAg and HCVAb was determined in all patients using enzyme immunoassay kits (FUJIREBIO, Tokyo, Japan). Based on the presence of serum antigens/antibodies, the patients were categorized into four groups: B-HCC (HBsAg positive), C-HCC (HCVAb positive), BC-HCC (Both HBsAg and HCVAb positive), and NBNC-HCC (both HBsAg and HCVAb negative). AIH was diagnosed based on the simplified diagnostic 
criteria proposed by the International Autoimmune Hepatitis Group [14] and PBC was diagnosed based on a PBC scoring system [15]. All NBNC-HCC patients, except those with AIH and PBC, were then further divided into two groups, the alcoholic liver disease group (ALD-HCC) and the non-alcoholic group (nonALD-HCC group) depending on alcohol consumption based on the following criteria: patients whose daily alcohol consumption over $80 \mathrm{~g}$ were included in the ALD-HCC group and the remaining patients were included in the nonALD-HCC group. We investigated the background characteristics between the two groups, including age, sex, diabetes, body mass index, hypertension, biochemical test results for liver function, Child-Pugh grade [16], tumor size, tumor number, portal invasion, TNM stage [17], and tumor markers at the time of diagnosis. The diagnosis of diabetes was based on the following criteria: random glucose $>200$ $\mathrm{mg} / \mathrm{dl}$ or fasting glucose $>126 \mathrm{mg} / \mathrm{dl}$, or hemoglobin A1c $>6.5 \%$ on two occasions. Hypertension was diagnosed when patients were pharmacologically treated for hypertension or if their arterial pressure was greater or equal to $140 / 90$.

The treatment for $\mathrm{HCC}$ was performed based on a consensus-based treatment algorithm for HCC proposed by the Japanese Society of Hepatology [2]. Hepatectomy (surgery) or local ablation (radiofrequency ablation; RFA) was performed for 3 or fewer nodules, if the nodules were $3 \mathrm{~cm}$ or smaller with no extrahepatic lesions, good liver function results, and no vascular invasion. Even if the number of nodules was 3 or fewer, if the tumor size exceeded $3 \mathrm{~cm}$, hepatectomy or transcatheter arterial embolization (TACE) was selected. For cases with 4 or more lesions, TACE or transcatheter arterial infusion (TAI) was selected, and resection was considered.

We compared the overall survival rates and cumulative recurrence rates after initial radical treatment between ALD-HCC and nonALD-HCC groups. We also studied the factors 
contributing to recurrence after initial remission and survival for both ALD-HCC and non ALD-HCC patients. Initial radical treatment (initial remission) was defined as follows: all of the HCC nodules (single/multiple) had disappeared following initial treatment, and no local recurrence or new tumors were detected on computed tomography within 6 months after the initial treatment.

\section{Statistical analysis}

Results are expressed as the mean values with standard deviation or the number (percentage) of patients with each variable. Comparison of background characteristics between ALD-HCC and nonALD-HCC patients was conducted using Fisher's exact test and Mann-Whitney U test. Overall survival rates and cumulative recurrence rates after initial remission were calculated using the Kaplan-Meier method and the differences between ALD-HCC and nonALD-HCC patients were examined by log rank test. The Cox proportional hazards model was used for multivariate analysis for factors that influenced survival and recurrence after the initial remission and performed separately for ALD-HCC and nonALD-HCC. Statistical data analysis was performed using the SPSS program, version 18.0 (SPSS, Chicago, IL). All reported P values were two-tailed, and statistical significance was set at $\mathrm{P}<0.05$.

\section{Results}

\section{Patients}

The 1109 HCC patients in our study comprised 177 NBNC-HCC (16\%), 127 B-HCC (11\%), $783 \mathrm{C}-\mathrm{HCC}(71 \%)$, and $22 \mathrm{BC}-\mathrm{HCC}(2 \%)$ patients. Of the $177 \mathrm{NBNC}-\mathrm{HCC}$ patients, 8 patients 
(4 diagnosed with AIH and 4 diagnosed with PBC) were excluded from the study. In addition, 42 other patients were excluded for the following reasons: natural death $(n=15)$, transfer to other hospitals $(n=18)$, and missing data $(n=9)$. We examined the detailed characteristics of the remaining 127 patients. Based on alcohol consumption, 42 patients were included in the ALD-HCC group and the other 85 patients were included in the nonALD-HCC group.

Clinical characteristics of patients with HCC: comparison between ALD-HCC and nonALD-HCC groups.

The clinical features of the 127 NBNC-HCC patients were investigated and compared between the ALD-HCC and nonALD-HCC groups (Table 1). Mean age at the time of diagnosis of HCC was significantly higher in nonALD-HCC patients than in the ALD-HCC group (71.4 vs. 66.4 , respectively; $\mathrm{P}<0.001)$. The proportion of men among nonALD-HCC patients was significantly lower than that among ALD-HCC patients (78\% vs. 93\%, respectively; $\mathrm{P}=0.034$ ). The prevalence of diabetes in the nonALD-HCC group was significantly higher than that in the ALD-HCC group (56\% vs. 33\%, respectively; $\mathrm{P}=0.015)$. Aspartate aminotransferase, alanine aminotransferase, and total bilirubin were significantly higher in the ALD-HCC group than in the nonALD-HCC group. In addition, Albumin levels and prothrombin time were significantly lower in the ALD-HCC group than in the nonALD-HCC group. Maximum tumor size in diameter $(\mathrm{cm})$ at the time of diagnosis was significantly larger (5.22 vs. 4.20 , respectively; $\mathrm{P}=0.007)$, and the proportion of patients with $\alpha$-fetoprotein levels greater than $100 \mathrm{ng} / \mathrm{ml}$ was significantly higher ( $32 \%$ vs. $12 \%$, respectively; $\mathrm{P}=0.014$ ) in the nonALD-HCC group than in the ALD-HCC group. The differences between groups in body mass index, hypertension, anti-HBc positivity, Child-Pugh grade, number of tumors, portal invasion, the TNM stage (I/II/III/IV), or 
des- $\gamma$-carboxy prothrombin were not significant.

\section{Treatment}

According to the consensus-based treatment algorithm for HCC proposed by the Japanese Society of Hepatology [2], initial treatment for NBNC-HCC patients was performed as follows: surgery $(n=7), \operatorname{RFA}(n=21)$, TACE/TAI $(n=12)$, and no treatment $(n=2)$ of the 42 ALD-HCC patients; and surgery $(n=32)$, RFA $(n=26)$, TACE/TAI $(n=26)$, and no treatment $(n=1)$ of the 85 nonALD-HCC patients. The proportion of patients receiving surgery as the initial treatment was significantly higher in the nonALD-HCC group than in the ALD-HCC group (38\% vs. $17 \%$, respectively; $\mathrm{P}=0.016$ ). The proportion of RFA was significantly lower in the nonALD-HCC group than in the ALD-HCC group (31\% vs. 50\%, respectively; $\mathrm{P}=0.033)$. In total, 22 of 42 ALD-HCC patients and 50 of 85 nonALD-HCC patients received initial radical treatment, and there was no significant difference ( $52 \%$ vs. $59 \%$, respectively; $\mathrm{P}=0.491$ ) between groups.

\section{HCC recurrence rates after the initial radical treatment.}

Of the 22 ALD-HCC patients with initial remission, recurrent HCC was detected in 14 patients during a median follow-up period of 28 months (range: 12-83 months). Of the 50 nonALD-HCC patients with initial remission, recurrent HCC was detected in 19 patients during a median follow-up period of 23 months (range: 9-81 months). The 5-year cumulative recurrence rates in ALD-HCC and nonALD-HCC patients were $69 \%$ and 52\%, respectively. Although the ALD-HCC group was considered to have higher recurrent tendency than the nonALD-HCC group, the difference was not statistically significant $(\mathrm{P}=0.304$, Figure 1$)$.

To identify the factors contributing to the cumulative recurrence of ALD-HCC and 
nonALD-HCC, we performed univariate analysis for several factors, but we detected no significant factor responsible for recurrence after the initial radical treatment (data not shown).

\section{Survival rates}

The 5-year survival rates of ALD-HCC and nonALD-HCC patients were $66 \%$ and $53 \%$, respectively, and the difference between groups was not significant ( $\mathrm{P}=0.352$, Figure 2$)$. To identify the factors contributing to the overall survival of ALD-HCC and nonALD-HCC patients, the Cox proportional hazards model was performed for multivariate analysis for factors that were considered to significantly contribute to survival on univariate analysis (Table 2). This analysis revealed that portal invasion of $\mathrm{HCC}$ was a significant factor for survival in ALD-HCC patients. On the other hand, a higher $\alpha$-fetoprotein level (over $100 \mathrm{ng} / \mathrm{ml}$ ) was a significant factor contributing to survival in nonALD-HCC patients.

\section{Discussion}

The number of patients with hepatic virus-unrelated HCC, i.e., NBNC-HCC, has been increasing annually in Japan $[3,5,8]$. Indeed, the current retrospective cohort study confirmed that the number and proportion of NBNC patients has increased in Osaka Red Cross Hospital. ; the incidence of NBNC-HCC patients among all HCC patients ranged from $12.8 \%$ in 2004 to 22.6\% in 2009 (data not shown). The clinical characteristics and prognosis of NBNC-HCC, however, have not been fully elucidated. Therefore, we studied in detail the clinical background and prognosis of a large number of NBNC-HCC patients in a single center and compared the clinical characteristics between those with alcohol-related and those with alcohol-unrelated HCC. 
The findings of the present study demonstrated that diabetes was significantly more frequent in the nonALD-HCC group than in the ALD-HCC group. An earlier large-scale epidemiologic study of 824,263 registered patients showed that among men with diabetes, the risk of HCC is doubled and this increase in risk is independent of alcoholic liver disease, viral hepatitis, or demographic features [18]. Another population-based study of 8244 patients reported that diabetes is associated with a 2 to 3 -fold increase in the risk of $\mathrm{HCC}$, regardless of the presence of other major HCC risk factors and that diabetes is an independent risk factor for HCC [19]. In Japan, the diagnosis of diabetes continues to increase. Indeed, between 1997 and 2007, people classified as "strongly suspected of having diabetes" increased from approximately 6.9 million to 8.9 million, and those classified as "people for whom the possibility of diabetes cannot be precluded" increased from approximately 6.8 million to 13.2 million [20]. This tendency may be associated with the increased incidence of NBNC-HCC patients.

The pathophysiology underlying the increased risk of HCC with diabetes is not certain. Diabetes is associated with NAFLD, including nonalcoholic steatohepatitis (NASH) with specific hepatic insulin resistance [21,22]. Insulin resistance facilitates peripheral lipolysis and the accumulation of free fatty acids in the liver, thus leading to NAFLD. Hepatocellular injury, inflammation, and, eventually, hepatic fibrosis can result in the occurrence of HCC [18]. A recent study indicated that diabetes-related NAFLD/NASH with elevated liver enzymes is associated with a clinically significant risk of developing end-stage liver disease, including HCC [23]. The annual NAFLD cumulative incidence of HCC is reported to be $2.6 \%$ in patients with NASH-cirrhosis [24]. Thus, in nonALD-HCC patients, a higher prevalence of diabetes could contribute to accumulating liver damage as NASH, and eventually HCC.

The diagnosis of NASH is based on following pathologic findings: hepatic steatosis, 
hepatocellular ballooning, lobular inflammation, pericellular or perisinuosoidal fibrosis, and Mallory body formation [25]. It is difficult to evaluate the role of hepatocyte fat deposition in the development of HCC, however, because the fat deposits in hepatocytes tend to disappear as liver fibrosis progresses; this phenomenon is referred to as burn-out NASH [26]. Indeed, based on histological analysis of tissues from hepatectomy, it was difficult to confirm pathologic evidence of NASH. In 6 patients with nonALD-HCC surgically treated, NASH-like pathologic findings, such as hepatic steatosis, hepatocellular ballooning, and Mallory body formation were identified, however, suggesting that some proportion of the nonALD-HCC group had NASH-based HCC.

In the present study, tumor size was larger, and tumor marker levels were higher in the nonALD-HCC group than in the ALD-HCC group. This is in part due to the lower opportunity of nonALD-HCC patients to undergo annual surveillance for chronic liver disease, and the tendency to be diagnosed with definite HCC at a more advanced stage. Surveillance has not been established for patients with NBNC-HCC because the risk factors are not well understood, other than excessive alcohol drinking. As a result, cryptogenic HCC patients, especially those with nonALD-HCC, are not detected until they reach an advanced stage [27]. Although the general consensus is that metabolic factors are related to the occurrence of $\mathrm{HCC}$, we have not established a method for determining the high risk group. Among patients with metabolic factors, more detailed examination of the possible carcinogenic factors is necessary for earlier detection of HCC.

Liver function results were altogether significantly worse in ALD-HCC patients than in nonALD-HCC patients. Because liver function in the ALD-HCC group was worse than that in the nonALD-HCC group, those in the ALD-HCC group were thought to undergo more liver injury, and this cumulative injury may increase the occurrence of HCC. Therefore, poor liver 
function could be the major reason of higher recurrence rate after initial remission of ALD-HCC than nonALD-HCC. As a result, ALD-HCC and nonALD-HCC patients had a similar prognosis. The ALD-HCC group was superior to the nonALD-HCC group in the tumor state, such as tumor size and tumor marker at the time of diagnosis, but inferior to the nonALD-HCC group in liver function, leading to recurrence. These good and bad points counteract each other and result in an equivalent prognosis between groups.

In conclusion, although the number of patients with NBNC-HCC has been increasing annually, many features of NBNC-HCC remain unknown. Based on the present study, the most common etiologic factor for NBNC-HCC was alcohol, and diabetes may be related to the occurrence of $\mathrm{HCC}$ in patients with nonalcohol-related liver disease. The comparison between groups revealed that nonALD-HCC tended to be detected at a more advanced stage whereas liver function in ALD-HCC was worse. Finally, the prognosis was equivalent between groups. Further examination of the risk factors for NBNC-HCC and establishing a precise surveillance system are needed to diagnose HCC earlier and develop curative therapies.

\section{References}

1 El-Serag HB, Marrero JA, Rudolph L, Reddy KR: Diagnosis and treatment of hepatocellular carcinoma. Gastroenterology 2008;134:1752-1763.

2 Kudo M, Okanoue T: Management of hepatocellular carcinoma in Japan: Consensus-based clinical practice manual proposed by the Japan Society of Hepatology. Oncology 2007;72 Suppl 1:2-15.

3 Hatanaka K, Kudo M, Fukunaga T, Ueshima K, Chung H, Minami Y, Sakaguchi Y, Hagiwara S, Orino A, Osaki Y: Clinical characteristics of NonBNonC- HCC: Comparison with HBV and HCV related HCC. Intervirology 2007;50:24-31.

4 Nordenstedt H, White DL, El-Serag HB: The changing pattern of epidemiology in hepatocellular carcinoma. Dig Liver Dis 2010;42 Suppl 3:S206-214.

5 Nouso K, Kobayashi Y, Nakamura S, Kobayashi S, Toshimori J, Kuwaki K, Hagihara H, Onishi H, Miyake Y, Ikeda F, Shiraha H, Takaki A, Iwasaki Y, Kobashi H, Yamamoto K: Evolution of prognostic factors in hepatocellular carcinoma in Japan. Aliment Pharmacol Ther 
2010;31:407-414.

6 Tanaka H, Imai Y, Hiramatsu N, Ito Y, Imanaka K, Oshita M, Hijioka T, Katayama K, Yabuuchi I, Yoshihara H, Inoue A, Kato M, Takehara T, Tamura S, Kasahara A, Hayashi N, Tsukuma H: Declining incidence of hepatocellular carcinoma in Osaka, Japan, from 1990 to 2003. Ann Intern Med 2008;148:820-826.

7 Nishiguchi S, Kuroki T, Nakatani S, Morimoto H, Takeda T, Nakajima S, Shiomi S, Seki S, Kobayashi K, Otani S: Randomised trial of effects of interferon-alpha on incidence of hepatocellular carcinoma in chronic active hepatitis $\mathrm{C}$ with cirrhosis. Lancet 1995;346:1051-1055.

8 Abe H, Yoshizawa K, Kitahara T, Aizawa R, Matsuoka M, Aizawa Y: Etiology of non-B non-C hepatocellular carcinoma in the eastern district of Tokyo. J Gastroenterol 2008;43:967-974.

9 Fattovich G, Stroffolini T, Zagni I, Donato F: Hepatocellular carcinoma in cirrhosis: Incidence and risk factors. Gastroenterology 2004;127:S35-50.

10 Hassan MM, Hwang LY, Hatten CJ, Swaim M, Li D, Abbruzzese JL, Beasley P, Patt YZ: Risk factors for hepatocellular carcinoma: Synergism of alcohol with viral hepatitis and diabetes mellitus. Hepatology 2002;36:1206-1213.

11 Kawaguchi T, Kakuma T, Yatsuhashi H, Watanabe H, Saitsu H, Nakao K, Taketomi A, Ohta S, Tabaru A, Takenaka K, Mizuta T, Nagata K, Komorizono Y, Fukuizumi K, Seike M, Matsumoto S, Maeshiro T, Tsubouchi H, Muro T, Inoue O, Akahoshi M, Sata M: Data mining reveals complex interactions of risk factors and clinical feature profiling associated with the staging of non-hepatitis B virus/non-hepatitis C virus-related hepatocellular carcinoma. Hepatol Res 2011;41:564-571.

12 Hashimoto E, Taniai M, Kaneda H, Tokushige K, Hasegawa K, Okuda H, Shiratori K, Takasaki K: Comparison of hepatocellular carcinoma patients with alcoholic liver disease and nonalcoholic steatohepatitis. Alcohol Clin Exp Res 2004;28:164S-168S.

13 Honda H, Ochiai K, Adachi E, Yasumori K, Hayashi T, Kawashima A, Fukuya T, Gibo M, Matsumata T, Tsuneyoshi M: Hepatocellular carcinoma: Correlation of CT, angiographic, and histopathologic findings. Radiology 1993;189:857-862.

14 Hennes EM, Zeniya M, Czaja AJ, Parés A, Dalekos GN, Krawitt EL, Bittencourt PL, Porta G, Boberg KM, Hofer H, Bianchi FB, Shibata M, Schramm C, Eisenmann de Torres B, Galle PR, McFarlane I, Dienes HP, Lohse AW, Group IAH: Simplified criteria for the diagnosis of autoimmune hepatitis. Hepatology 2008;48:169-176.

15 Yamamoto K, Terada R, Okamoto R, Hiasa Y, Abe M, Onji M, Tsuji T: A scoring system for primary biliary cirrhosis and its application for variant forms of autoimmune liver disease. J Gastroenterol 2003;38:52-59.

16 Pugh RN, Murray-Lyon IM, Dawson JL, Pietroni MC, Williams R: Transection of the oesophagus for bleeding oesophageal varices. Br J Surg 1973;60:646-649.

17 Greene FL PD, Fleming ID et al.: AJCC cancer staging manual, 6th edition. Chicago:Springer 2002

18 El-Serag HB, Tran T, Everhart JE: Diabetes increases the risk of chronic liver disease and hepatocellular carcinoma. Gastroenterology 2004;126:460-468.

19 Davila JA, Morgan RO, Shaib Y, McGlynn KA, El-Serag HB: Diabetes increases the risk of hepatocellular carcinoma in the United States: A population based case control study. Gut 2005;54:533-539.

20 Morimoto A NR, Tajima N: Trends in the epidemiology of patients with diabetes in 
Japan. JMAJ 2010;53:36-40.

21 Marchesini G, Brizi M, Bianchi G, Tomassetti S, Bugianesi E, Lenzi M, McCullough AJ, Natale S, Forlani G, Melchionda N: Nonalcoholic fatty liver disease: A feature of the metabolic syndrome. Diabetes 2001;50:1844-1850.

22 Marchesini G, Bugianesi E, Forlani G, Cerrelli F, Lenzi M, Manini R, Natale S, Vanni E, Villanova N, Melchionda N, Rizzetto M: Nonalcoholic fatty liver, steatohepatitis, and the metabolic syndrome. Hepatology 2003;37:917-923.

23 Ekstedt M, Franzén LE, Mathiesen UL, Thorelius L, Holmqvist M, Bodemar G, Kechagias S: Long-term follow-up of patients with NAFLD and elevated liver enzymes. Hepatology 2006;44:865-873.

24 Ascha MS, Hanouneh IA, Lopez R, Tamimi TA, Feldstein AF, Zein NN: The incidence and risk factors of hepatocellular carcinoma in patients with nonalcoholic steatohepatitis. Hepatology 2010;51:1972-1978.

25 Matteoni CA, Younossi ZM, Gramlich T, Boparai N, Liu YC, McCullough AJ: Nonalcoholic fatty liver disease: A spectrum of clinical and pathological severity. Gastroenterology 1999;116:1413-1419.

26 Poonawala A, Nair SP, Thuluvath PJ: Prevalence of obesity and diabetes in patients with cryptogenic cirrhosis: A case-control study. Hepatology 2000;32:689-692.

27 El-Serag HB, Rudolph KL: Hepatocellular carcinoma: Epidemiology and molecular carcinogenesis. Gastroenterology 2007;132:2557-2576. 


\section{【Headings of Tables】}

\section{Table 1.}

Clinical characteristics of nonBnonC-HCC

\section{Table 2.}

Factors associated with survival according to Cox proportional hazard analysis

\section{【legends of Figures】}

\section{Fig. 1.}

Cumulative recurrence rates after initial remission. The 5-year cumulative recurrence rates in ALD-HCC and nonALD-HCC patients were 69\% and 52\%, respectively. Although the ALD-HCC group was considered to have higher recurrent tendency than the nonALD-HCC group, the difference was not statistically significant $(\mathrm{P}=0.304)$.

\section{Fig. 2.}

Overall survival rates. The 5-year survival rates of ALD-HCC and nonALD-HCC patients were $66 \%$ and 53\%, respectively, and the difference between groups was not significant (P $=0.352$ ). 
Soo Ki Kim et al.

Table 1. Clinical characteristics of nonBnonC-HCC

\begin{tabular}{|c|c|c|c|}
\hline Group & $\begin{array}{l}\text { alcohol } \\
\mathrm{N}=42\end{array}$ & $\begin{array}{l}\text { non-alcohol } \\
\mathrm{N}=85\end{array}$ & $\begin{array}{l}\text { Statistics } \\
\mathrm{P} \text { value }\end{array}$ \\
\hline Age & $66.4 \pm 7.6$ & $71.4 \pm 9.1$ & $<0.001$ \\
\hline Sex(Male/female) & $39 / 3$ & $66 / 19$ & 0.034 \\
\hline $\mathrm{DM}(+/-)$ & $14 / 28$ & $48 / 37$ & 0.015 \\
\hline $\mathrm{BMI}(>25 / \leqq 25)(\%)$ & $41 / 59$ & $41 / 59$ & NS \\
\hline $\mathrm{HT}(+/-)$ & $13 / 29$ & $35 / 50$ & NS \\
\hline $\operatorname{HBcAb}(+/-)$ & $24 / 18$ & $37 / 48$ & NS \\
\hline AST(IU/L) & $59.9 \pm 35.5$ & $50.9 \pm 49.7$ & $<0.001$ \\
\hline ALT(IU/L) & $44.8 \pm 33.3$ & $35.9 \pm 34.6$ & 0.042 \\
\hline $\mathrm{T}-\mathrm{Bil}(\mathrm{mg} / \mathrm{dl})$ & $1.12 \pm 0.65$ & $0.92 \pm 0.64$ & 0.020 \\
\hline $\mathrm{Alb}(\mathrm{g} / \mathrm{dl})$ & $3.72 \pm 0.54$ & $3.92 \pm 0.51$ & 0.022 \\
\hline $\mathrm{PT}(\%)$ & $81.4 \pm 21.8$ & $90.3 \pm 20.3$ & 0.037 \\
\hline Child-Pugh grade(A/B/C) & $28 / 13 / 1$ & $67 / 14 / 4$ & $\mathrm{NS}$ \\
\hline Maximum tumor size $(\mathrm{cm})$ & $4.20 \pm 3.67$ & $5.22 \pm 3.88$ & 0.007 \\
\hline Number of tumors(single/multiple) & $21 / 21$ & $50 / 35$ & $\mathrm{NS}$ \\
\hline Portal invasion $(+/-)$ & $5 / 37$ & $13 / 72$ & NS \\
\hline TNM Stage(I/II/III/IV) & $10 / 16 / 11 / 5$ & $6 / 43 / 23 / 13$ & $\mathrm{NS}$ \\
\hline $\operatorname{AFP}(>100 / \leqq 100)(\mathrm{ng} / \mathrm{ml})$ & $5 / 37$ & $27 / 58$ & 0.014 \\
\hline $\mathrm{DCP}(>100 / \leqq 100)(\mathrm{mAu} / \mathrm{ml})$ & $26 / 16$ & $58 / 27$ & NS \\
\hline
\end{tabular}

NS:not significant. 
Soo Ki Kim et al.

Table 2. Factors associated with survival according to Cox proportional hazard analysis

\begin{tabular}{lllll}
\hline Group & alcohol & & non-alcohol \\
& $\mathrm{N}=42$ & & & \\
\hline Risk factors & Hazard ratio (95\% CI) & $\mathrm{p}$ & Hazard ratio (95\% CI) & $\mathrm{p}$ \\
\hline Maximum tumor Size $>3 \mathrm{~cm}$ & $\mathrm{NS}$ & $\mathrm{NS}$ & $\mathrm{NS}$ & $\mathrm{NS}$ \\
Portal invasion(+) & $11.9(0.90-157.4)$ & 0.05 & $\mathrm{NS}$ & $\mathrm{NS}$ \\
Tumor stage III/IV & $\mathrm{NS}$ & $\mathrm{NS}$ & $\mathrm{NS}$ & $\mathrm{NS}$ \\
AFP $>100 \mathrm{ng} / \mathrm{ml}$ & $\mathrm{NS}$ & $\mathrm{NS}$ & $4.63(1.57-13.6)$ & 0.005 \\
DCP $>100 \mathrm{mAU} / \mathrm{ml}$ & $\mathrm{NS}$ & $\mathrm{NS}$ & $\mathrm{NS}$ & $\mathrm{NS}$ \\
\hline
\end{tabular}

NS:not significant. 


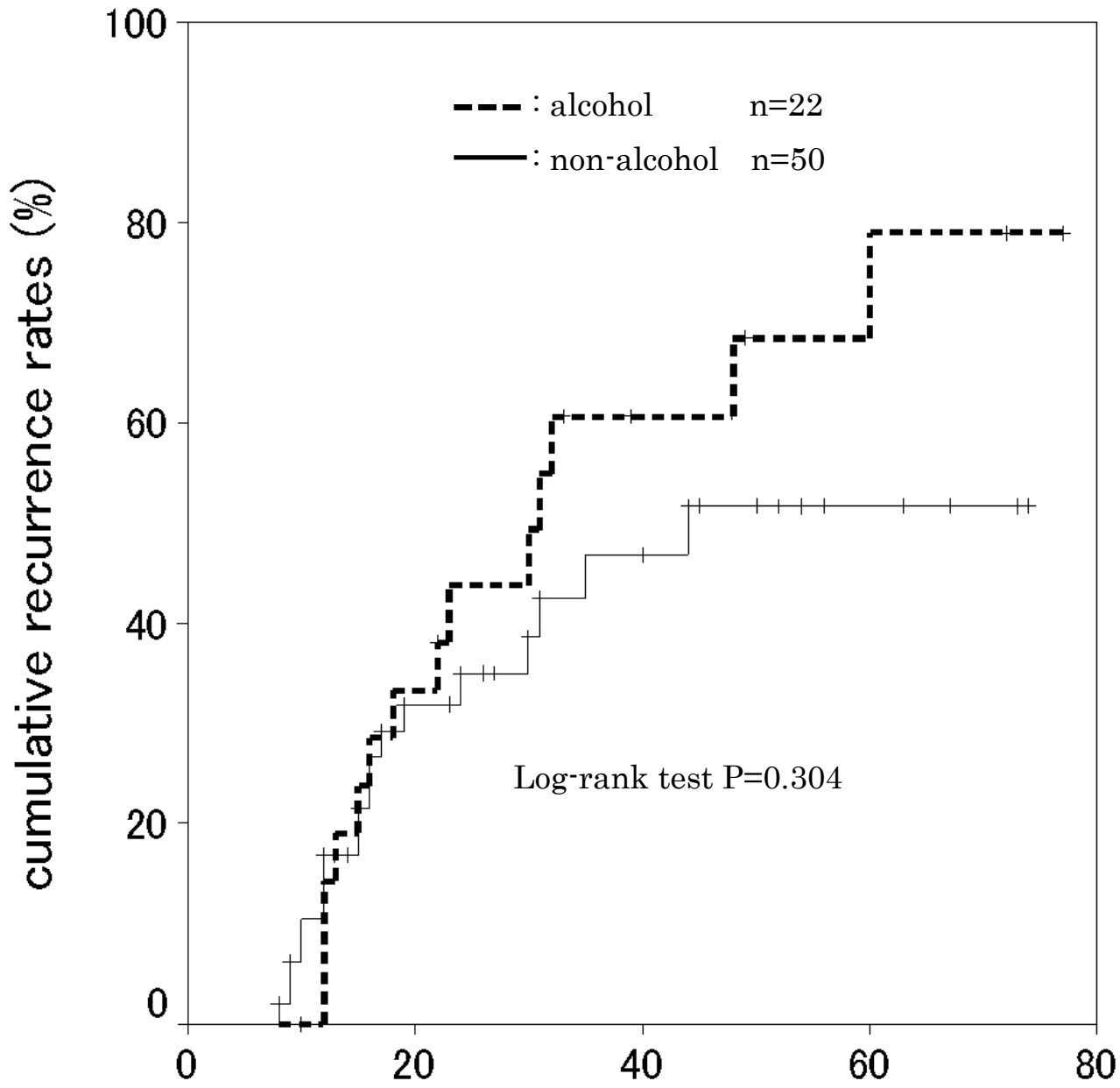

\section{Months}

Fig. 1. Cumulative recurrence rates after initial remission. The 5-year cumulative recurrence rates in ALD-HCC and nonALD-HCC patients were 69\% and $52 \%$, respectively. Although the ALD-HCC group was considered to have higher recurrent tendency than the nonALD-HCC group, the difference was not statistically significant $(\mathrm{P}=0.304)$.

Soo Ki Kim et al. 


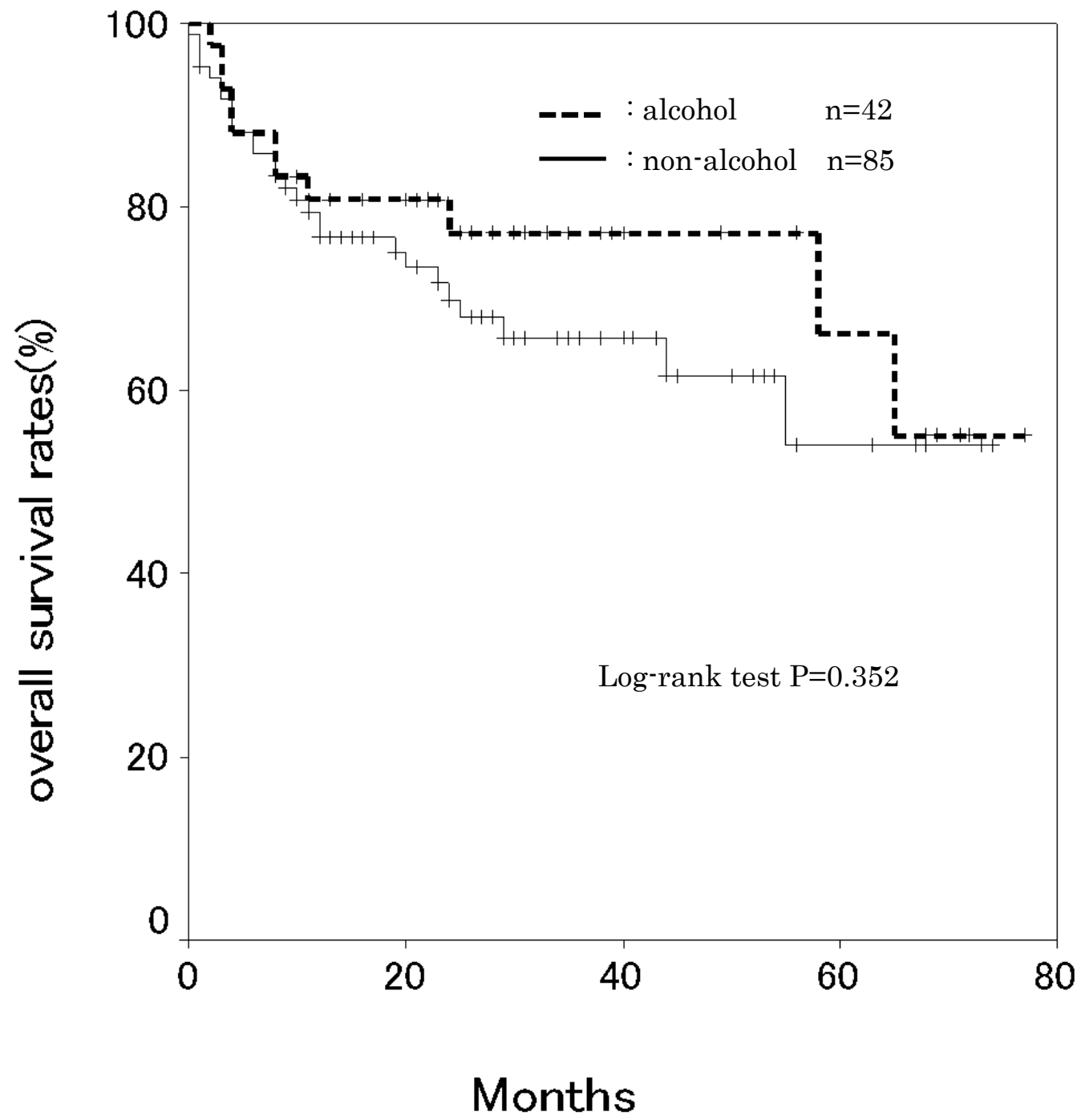

Fig. 2. Overall survival rates. The 5-year survival rates of ALD-HCC and nonALD-HCC patients were $66 \%$ and $53 \%$, respectively, and the difference between groups was not significant $(\mathrm{P}=0.352)$.

Soo Ki Kim et al. 